\title{
Deep Learning and Artificial Intelligence: Project Collaboration across Classes
}

\section{Prof. Franz J Kurfess, California Polytechnic State University, San Luis Obispo}

Franz J. Kurfess is a professor in the Computer Science and Software Engineering Department, California Polytechnic State University, San Luis Obispo, where he teaches mostly courses in Artificial Intelligence, Human-Computer Interaction, and User-Centered Design. Before joining Cal Poly, he was with Concordia University in Montreal, Canada, the New Jersey Institute of Technology, the University of Ulm, Germany, the International Computer Science Institute in Berkeley, CA, and the Technical University in Munich, where he obtained his M.S. and Ph.D. in Computer Science.

His main areas of research are Artificial Intelligence and Human-Computer Interaction, with particular interest in the intersection of the two fields.

\section{Prof. Maria Pantoja, CalPoly San Luis Obispo}

Maria Pantoja Computer Engineering Computer Science \& Software Engineering Office: 14-211 Phone Number: 805-756-1330 Email: mpanto01@calpoly.edu Homepage: https://cpe.calpoly.edu/faculty/mpanto01/ Biography B.S., Universidad Politecnica de Valencia, Spain Ph.D., Santa Clara University

Research Interests High Performance Computing Neural-Electronics Parallel Computing

\section{Dr. Irene Humer, California Polytechnic State University San Luis Obispo}

$\mathrm{Ph}$. D. Electrical Engineering and Information Technology, Vienna University of Technology M. S. Physics, University of Vienna M. S. Education Physics and Mathematics, University of Vienna

Research Interests: Computer Science Education, Physics Simulation, Applied Computing 


\title{
Deep Learning and Artificial Intelligence:
}

\section{Project Collaboration across Classes}

\begin{abstract}
Working in collaborative environments is an essential skill for computing professionals. In our program, students have significant team experience from previous classes; almost all of our classes in Cal Poly's Computer Science, Software Engineering and Computer Engineering programs have lab sections, and students start with team-based work early. However, they mostly work within small teams of about five members that are stable throughout a term. To stretch the students' collaborative skills and enhance their experience with more fluid team configurations, we experimented with creating teams across two related senior-level classes within Computer Science, but with different perspectives and approaches. For one particular project, shark spotting in drone video footage, the teams also collaborated with a group of Marine Biologists from the Shark Lab at CSU Long Beach, who provided the video material and acted as a customer for three teams across the two classes. This exposed our students to collaborators among different fields, with their own terminology, goals, work methods and practical approaches. Our paper reports on the initial experiment during the Fall 2019 term, involving two sections of an Artificial Intelligence class and one section of a Deep Learning class. We are planning to continue this collaboration in the future.
\end{abstract}

Keywords: Collaborative Learning, Interdisciplinary, Inter-Class teamwork

\section{Introduction}

Students at California Polytechnic State University, San Luis Obispo (Cal Poly) are exposed to practical, hands-on educational activities throughout their course of studies, reflected by the university's "Learn by Doing" motto. In the Computer Science, Software Engineering and Computer Engineering programs, almost all classes have lab components, and many of those lab activities involve teamwork. The introduction several years ago of a pre-programming class, CSC 123 Introduction to Computing, where students work in teams on projects focusing on computational thinking skills, has brought significant improvements in student attitudes, retention (especially of underrepresented groups), and collaboration. This class is taught in multiple sections of about 30 students, with different themes like Computing and Interactive Arts, Games Development, Robotics, or Mobile Development.

Collaborative learning is an educational approach to teaching and learning that involves groups of learners working together to solve a problem, complete a task or create a product [1]. Research shows very clear benefits of implementing group activities and group projects [1,2,3] for learning, including in higher education. In addition, hands-on applied teamwork exercises have been shown to benefit all students and female students in particular [6]. Collaborative environments also prepare students for work in the industry, where companies expect employees to work well with others and to communicate with other members of their professional teams and teams in other departments. We, as educators, sought to design authentic cross-class group experiences to help students practice working in teams and experience the relationship of two 
closely connected but distinct fields of computer science. Our collaboration involves two distinct but related courses in Computer Science: Deep Learning (DL) and Artificial Intelligence (AI). While both class contents are unique, Deep Learning can be considered as a subset of AI, and DL is heavily studied and used in AI. In addition, many of the very interesting problems in AI are currently being solved using DL. We felt an inter-class experience would allow students to practice working on a hands-on project as a team, similar to what they are likely to experience in industry.

There is a significant number of articles written on the benefits of research collaborations to improve the retention of minority students and faculty [18]; [19] a seminar series for high school juniors and seniors is described. The seminars ran for five years and students were exposed to research collaborations in different STEM fields.

Undergraduate research experiences have been shown to increase confidence, sense of belonging and prove a pathway to a scientific career for minority students, and the data indicate that most of these students intend to continue on this path [20, 21, 22]. In addition to engaging students in research projects, the likelihood of women and minorities to continue in a scientific discipline is further increased if the problems have a valuable connection to society [23, 24]. Our real-world project topics were of high interest to the students who appreciated their practical, scientific and environmental importance. The cross-course disciplinary setup encouraged teamwork and enhanced communication as well as social skills, breaking down certain stereotypes about computer science [25] and increasing the retention of underserved minorities.

To enhance the team-based experiences and skills of the students, two instructors of senior-level courses with some overlap in content, M. Pantoja and F. Kurfess, engaged in an experiment to coordinate project activities across two of their courses. The data collection and analysis was coordinated by I. Humer, a colleague with a significant educational background and not involved in the teaching of the two classes. Our intent underlying this inter-class collaboration was threefold: first, to expose the students to more realistic collaboration conditions they are likely to encounter in industry; second, to build up and apply expertise in a real-world problem from a different domain; and third, to enhance their understanding and retention of knowledge and skills through interactions with other teams and external partners. This experiment fits into a larger set of efforts engaging students across multiple classes within the same field, across different disciplines, and within larger frameworks such as cross-disciplinary minors in our department.

During the Fall 2019 term, students from two classes in the Computer Science and Software Engineering Department, "Deep Learning" (taught by M. Pantoja) and "Introduction to Artificial Intelligence" (taught by F. Kurfess) selected project topics from a list created by the two instructors. Teams with related topics coordinated their activities to varying degrees, depending on the overlap of data sets, methods and tools, as well as practical considerations such as room size and class time constraints.

In the example project we will focus on here, "Shark Spotting with Drones," teams working on detecting and tracking sharks in drone aerial images developed three different approaches to identify sharks in aerial video footage available through a collaboration with marine biologists from the Shark Lab at CSU Long Beach. While the high-level contact was between the faculty at the two institutions, the student teams were directly involved in the curation of the overall data 
set collected by Marine Biology graduate students, the implementation of solutions and the validation methods. The focus for the teams was on the identification of frames in videos with objects of interest, primarily sharks. This required image segmentation, and the categorization into different objects such as sharks, seals, and surfers. The long-term goal of this project is twofold: modeling of shark behavior, such as shark herds (groups of sharks), and identifying possible interactions between sharks and other entities such as seals or surfers. The latter, in particular, can then be expanded to provide real-time warnings of shark presence at beaches. A more pragmatic objective was to reduce the amount of time spent by Marine Biology students watching drone footage video. Most of this footage shows water surface with no objects of interest. Thus, a system that recognizes objects of interest in the footage and marks those segments, would greatly reduce that time. However, since this was the first set of teams to tackle this problem, we did not expect a usable system from the class projects. An expansion of the shark spotting approaches described here is the subject of an ongoing Master's thesis at Cal Poly.

To evaluate the collaboration, we use the shark team collaboration project as a basis, since we had more teams in each class working on this particular project. We use the quality of the work produced by the student teams as well as their feedback on the collaboration as main assessment criteria. The results are summarized in Section 3.

In general, the collaboration was structured with the two instructors jointly leading the inter-class collaboration. During the first week, we presented the main projects to the students in both classes. In Week 4, students from both classes had a conference call with the Shark Lab marine biologist clients to clarify the task they needed to solve. Individual teams and students communicated directly with graduate students from the Shark Lab. While we considered a class trip to the Shark Lab facilities, the combination of time constraints, expenses, and logistics turned out to be too complex.

In this article, we present a detailed description of the implementation of the inter-class collaboration, the results of the collaboration via lab results and student surveys, our conclusions, and ongoing work related to this collaboration.

\section{Implementation}

The two courses involved in this inter-class collaboration are both upper-division computer science technical electives. The AI class consisted of two sections, the DL class of one. Each section had close to 40 students enrolled. For the collaboration, teams consisted of 2-6 students. Students could select their own teams, and teams could choose their own topics subject to approval by the respective instructor. The instructors provided a common list of suggested topics for both classes; about half of the teams chose topics from that list. The lab portion of the collaboration spanned the entirety of the academic term (a quarter with ten weeks of instruction). In the first week, students chose a problem they wanted to work on, followed by a group communication with the clients for the project. In Week 4 or 5, the teams had to explain their solution to the other teams, either in a presentation or as a science-fair style project display.

As an immediate goal for the shark project, the clients at the Shark Lab mainly wanted to identify the start and end times of snippets of video with the presence of sharks on video footage taken with a drone. They expressed very clearly that it would be very useful to them to mark at 
what time there is a shark occurrence in the video. The current solution they are using has a lot of false positives confusing sharks with dolphins, seals, surfers or kayaks. Their ultimate goal is to study the behavior of sharks, and simply identifying pieces of footage with shark appearances is a great help since it reduces the need to manually extract those snippets from the overall drone video footage. A further goal for them is to create models of shark behavior from video footage.

In general, this is a classification problem and can be tackled with feature extraction techniques. Since 2012 most solutions to image classification problems have been solved using Deep Learning methods, with much better performance than classical, hand-coded approaches. While the shapes of sharks and dolphins, for example, are distinct in high-quality images, in the lowquality drone video footage at our disposal they appear similar. For experts it is easy to differentiate between one and the other even in low-quality footage by analyzing the movement trajectories: sharks move their tails sideways, while dolphins often jump up and down. This information can be extracted from the video, but in the short time frame the teams had available it was not realistic to achieve this.

The teams chose different solutions, although all of them are based on Deep Learning:

- Team 1. Before training their network, this team from the DL class created a standardized dataset containing aerial images of sharks. They started by extracting still frames from a collection of drone footage of sharks taken from data provided by the Shark Lab group and additional video sources from the Internet. The group pulled frames at constant time intervals (e.g., 1 frame/sec) as opposed to consecutively to avoid having similar and thus redundant images in our dataset. Then, the team cropped each image into a square such that it contained all sharks in their entirety and downsized the resulting image to 512 x 512 pixels. They labeled and bounded each shark in each image using a labeling software. The resulting dataset consisted of 1,674 images of sharks shown from above. Of those, 1,422 were used for training and 252 were used for testing. They used heavy data augmentation including artificially adding fog. To detect sharks, they used both classification and object detection with bounding boxes to determine if and where a shark is present in the drone footage. The added step of object detection helps visualize the location of a shark and has a couple of advantages. First, it allows users to verify the network's claim and rule out any false positives. Second, with the knowledge of a shark's location, authorities and supervisors such as lifeguards could make more-informed choices when handling dangerous situations. They determined the bounding boxes of sharks in images using a Faster R-CNN network architecture [8-13].

- Team 2. This team from the AI class extracted still images of sharks from the video collection and performed semantic segmentation to separate objects from the background. Based on labeled training data, their model then outlined objects of interest in the videos. Their primary interest was in classifying those objects as sharks, surfers, or "other", including images that have multiple objects. In images with both sharks and surfers, they calculated the relative location of sharks and surfers and their likely trajectories in order to predict possible encounters. With ImageAI as their base library [14], they used TensorFlow, OpenCV, and Keras for their implementation. Through data augmentation techniques, they increased their pool of labeled images from 700 to 4200 . Their final model detected sharks with 90 - 98\% accuracy, and surfers with higher than $80 \%$. 
- Team 3. Also from the AI class, this team attempted to detect and identify sharks from video images, distinguish between sharks, other marine life and surfers in the images, and identify groups of sharks. The team used the YOLOv3 object detection neural network [16] to train their model using the same set as Team 2 of about 700 labeled images. They used data augmentation methods included in the YOLOv3 library but did not report the size of the augmented training set. The final result of the system is footage with sharks, where shark objects are marked with bounding boxes and the probability that the network believes the object is a shark.

The final deliverable for all teams in the two classes were conference-style research papers, with sections for previous work, implementation details and results. Due to the time constraints and the lack of technical knowledge especially for the AI class teams, each report on its own is not quite up to conference submission standards; however, since the teams chose somewhat different approaches, we are currently preparing a joint report for all teams.

\section{Results}

Our initial intention was to compare the quality of the three projects based on their final reports, with a commonly used template for conference submissions as the unifying framework. However, we did not succeed in imposing a common way to present the performance results of the models the teams implemented. In hindsight, we should have given the students clear instructions on the ways to report these results. As is fairly typical for such class projects, much of the work was done in the final weeks and days of the quarter, and clearly the students had other priorities than following our requests for a common format for their results. Since we have access to the data set and the codebases of the teams, we were able to run another set of experiments to obtain a limited comparable basis for reporting the results from the work of Team 2 ; we have not been successful so far with the work of Team 3. In the next iteration of this collaboration, we will give the students better instructions in this regard.

For each team, we will present the main results, together with sample images from their data sets.

- Team 1: This team reported results that are consistent with previously published work on similar projects. Their primary evaluation criterion is the degree of overlap between the object bounding box from a reference image, and the bounding box identified by the network. Ideally, this overlap would be $100 \%$, but this is neither practical nor necessary. From a practical perspective, mean average precision with a $50 \%$ overlap of the bounding boxes is useful in detecting relevant objects, while still giving reasonable performance. Increasing the overlap to $75 \%$ leads to a significant drop in performance.

\begin{tabular}{l|ccc|} 
Data Set & $\boldsymbol{m A P}_{25}$ & $\boldsymbol{m A P}_{50}$ & $\boldsymbol{m A P}_{75}$ \\
\hline Training (1,422 images) & 0.910 & 0.662 & 0.072 \\
Test (252 images) & 0.911 & 0.673 & 0.074
\end{tabular}

Table 1. Mean Average Precision of the network is evaluated based on the overlapping area of the actual bounding box and the predicted bounding box divided by the sum of both boxes' area. Precision was sampled at $25 \%, 50 \%$, and $75 \%$ overlap [26]. 

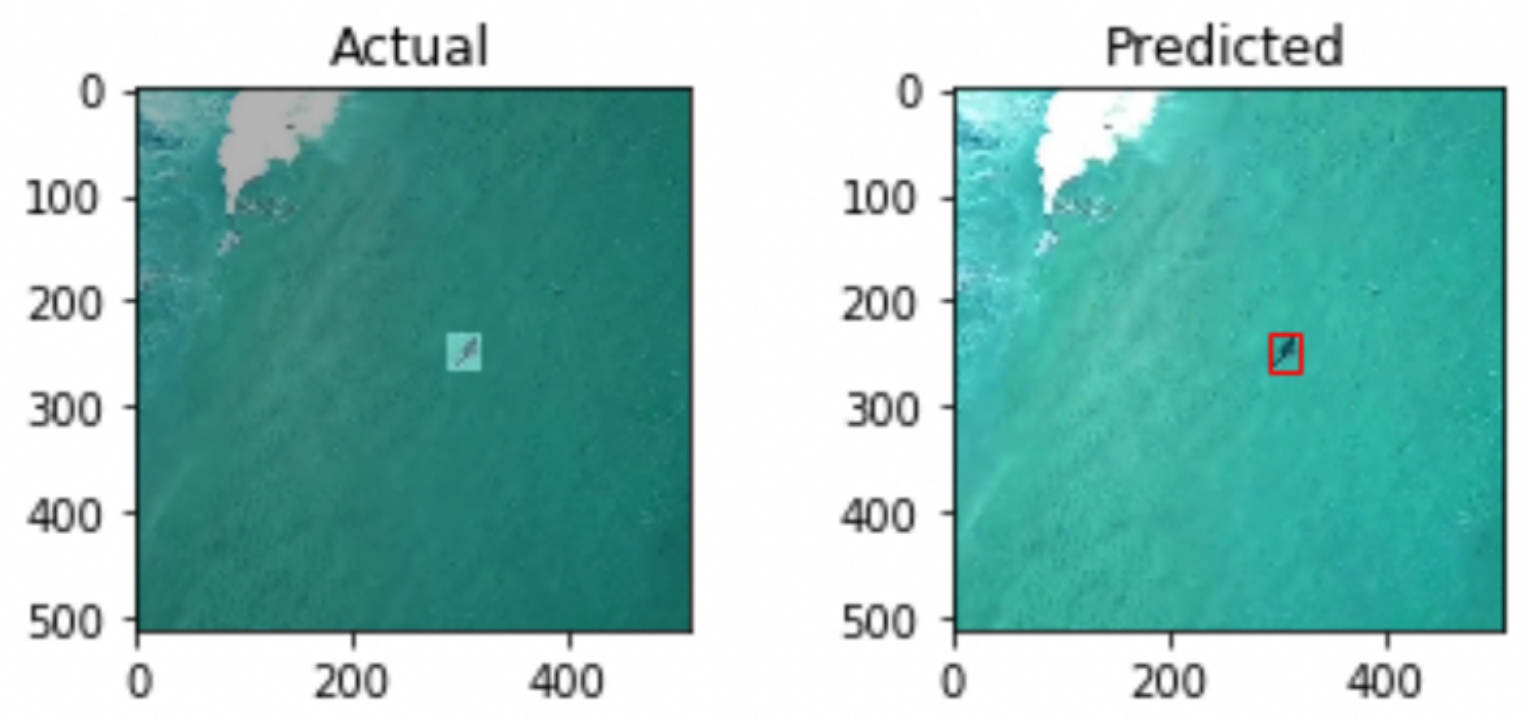

Fig. 2. Detector working on a video with artificial fog added and on the original video [26].

- Team 2: Their final model detected sharks with 90 - 98\% accuracy, and surfers with higher than $80 \%$. The training was conducted over multiple sessions due to computational resource limitations; as a consequence, mean average precision data are available only for the test set. Their results are in the same range as Team 1.

\begin{tabular}{|l|l|l|l|l|l|}
\hline Data Set & \# Images & Classes & $\mathrm{mAP}_{25}$ & $\mathrm{mAP}_{50}$ & $\mathrm{mAP}_{75}$ \\
\hline Test & 368 & sharks, surfers, boats, person & 0.89 & 0.64 & 0.070 \\
\hline
\end{tabular}

Table 2: Mean Average Precision, based on the overlapping areas of the actual bounding box and the predicted bounding box, divided by the sum of the two, and sampled at 25\%, 50\%, and $75 \%$ overlap [27]. 


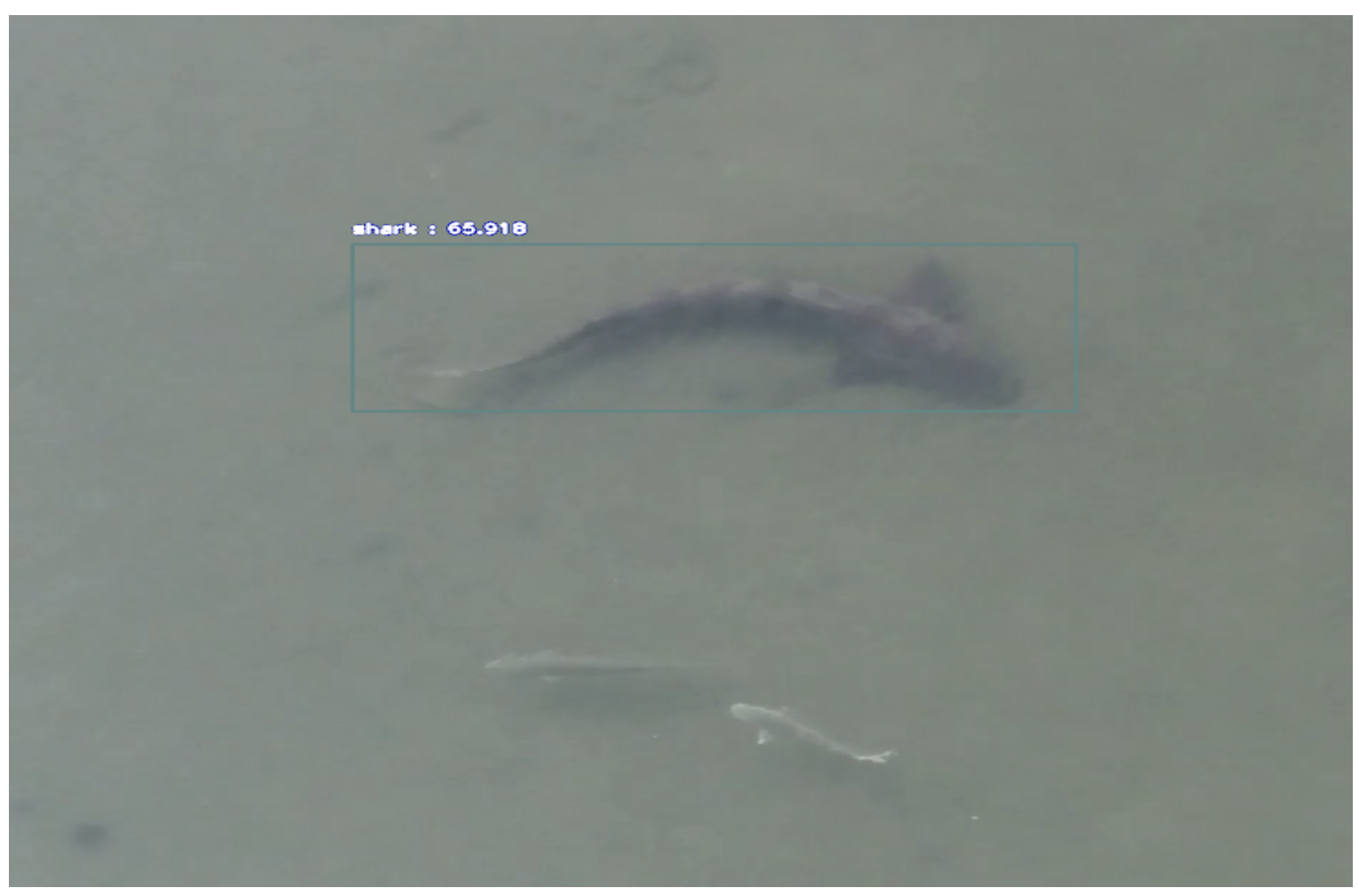

Fig. 3. Detection of an individual leopard shark with $65.918 \%$ probability. Two other sharks were not detected [27].

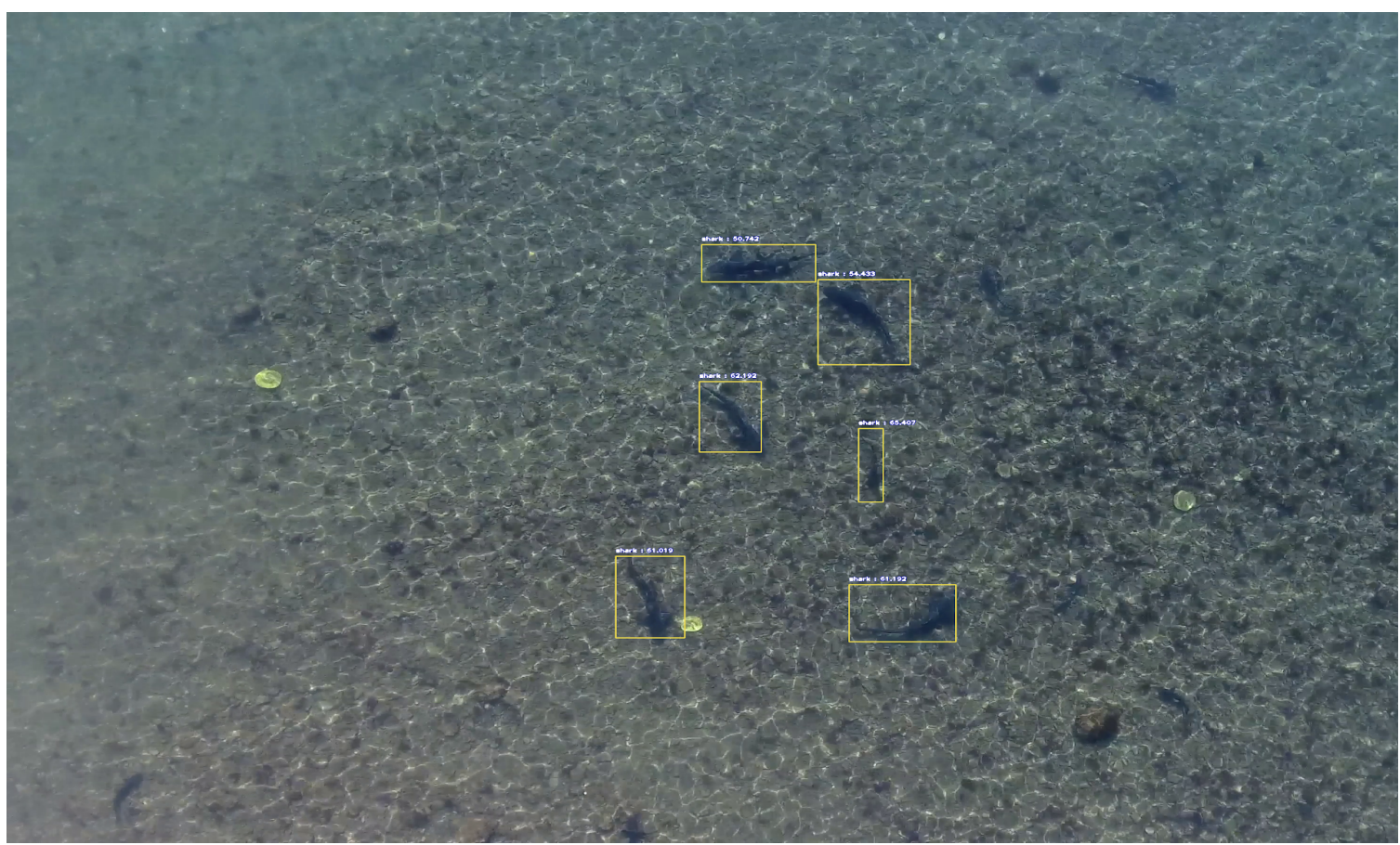

Fig. 4. Detection of multiple sharks in a herd; other objects likely to be sharks undetected [27]. 
- Team 3: The mean average precision at 50\% overlap reported by this team is low, at 0.113 . One reason communicated by the team was the lack of time for further training at the end of the quarter.

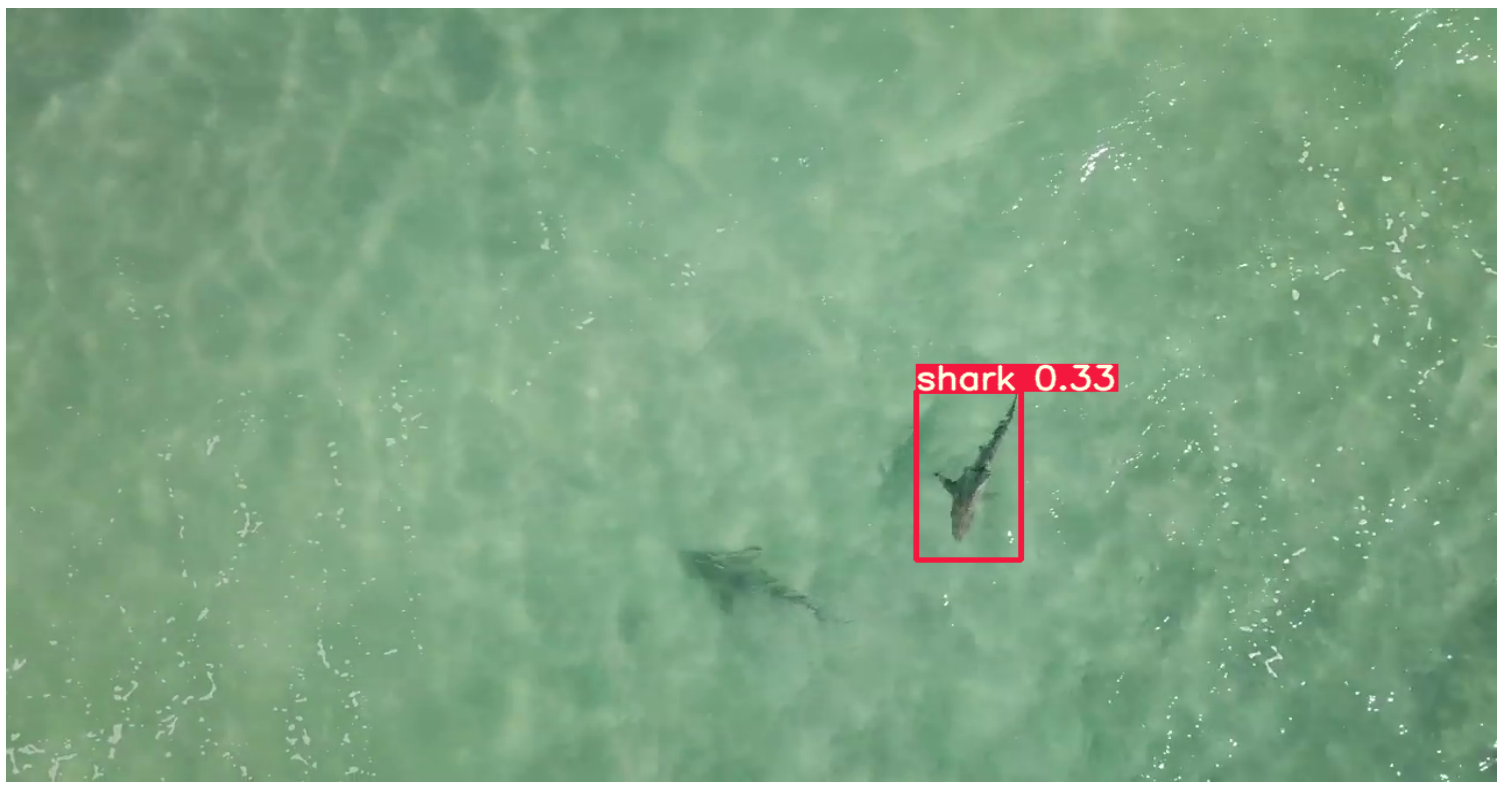

Fig. 5. Detection of an individual shark with 33\% probability, while another shark is not detected [27].

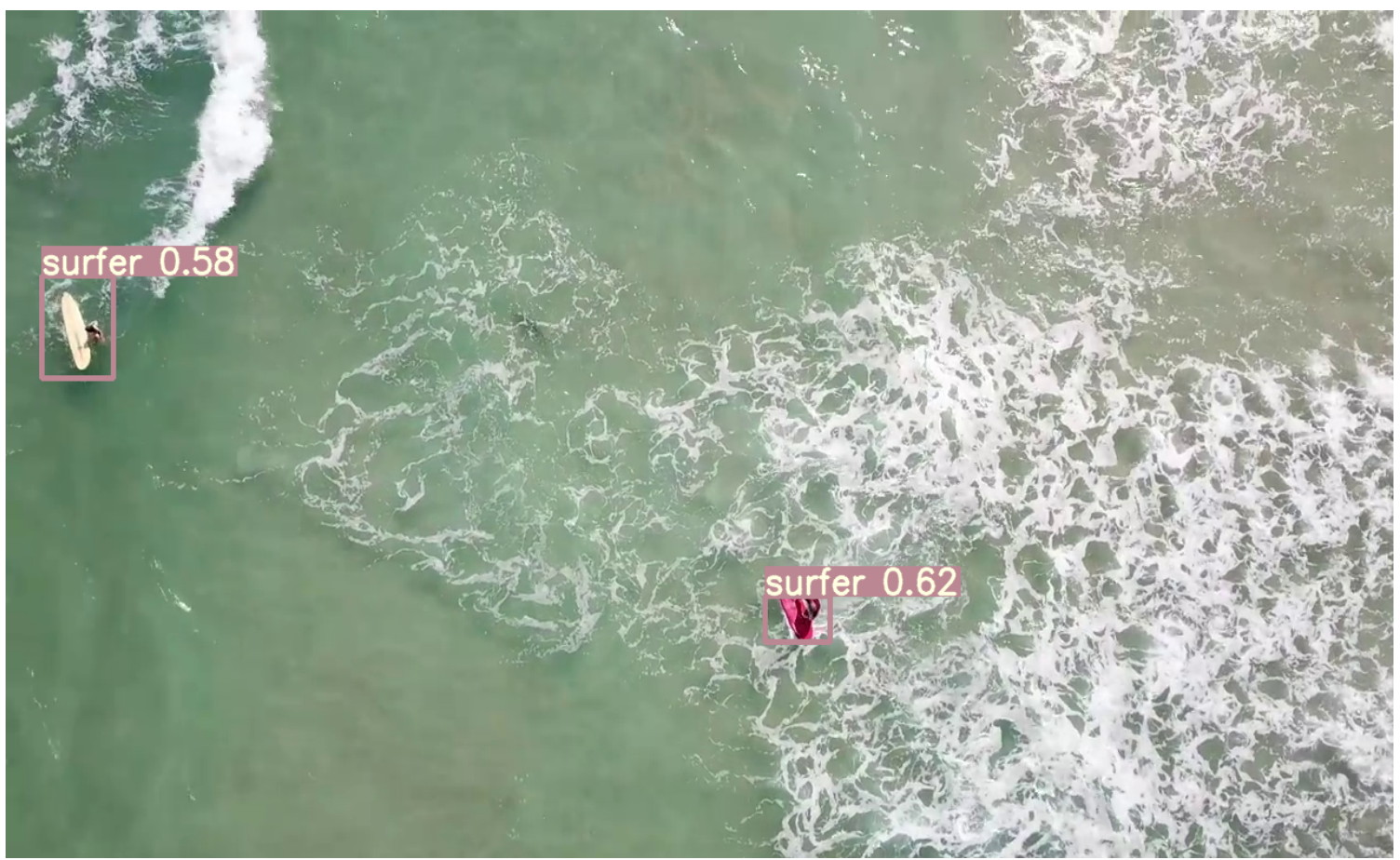

Fig. 6. Detection of two surfers. One object that may be a shark is not detected. Visibility is impacted by waves and foam [27]. 
- Collaboration Across Teams: The collaboration of the three teams did not meet our expectations. The two teams from different sections of the AI class taught by the same instructor were in reasonably close contact and kept each other informed about their progress and activities. This did not work so well with the team from the DL class. While the instructors walked the teams from the different classes to several joint meetings and initiated the setup of a communication infrastructure via Slack, GitHub, and a shared data repository, without direct intervention from the instructors the teams did not communicate. In the end, we did succeed at getting three different implementations for the same problem. All three teams succeeded in identifying the subjects (sharks) using the same input data and instructions from the client. And although not all teams reported their results in a directly comparable format, the overall outcome of their work confirms that it is possible to use Deep Learning methods to identify sharks in drone video footage. The results from the AI class also indicate that passable outcomes can be delivered by teams without prior knowledge of Deep Learning within a relatively short time frame.

\section{Educational Experience.}

In the course of the quarter during which this collaboration took place, some teams established solid foundations for collaboration across the classes and with their external partners. Others required some encouragement and guidance, but all teams showed a very positive attitude towards this effort. However, there were some logistical difficulties: of the three sections involved (two from the AI class, one from the Deep Learning class), there was an overlap between the lecture time of one AI class and the lab time of the DL class. The lectures and lab locations were in different, but close by, buildings and the rooms are not large enough to hold students from more than one class. In addition, the timeline for the class activities was different across the two classes: the AI classes started working on the project in Week 2, while the DL class focused on other assignments initially, and on the team project during the last few weeks of the quarter. To overcome the logistical issues, the instructors walked with the teams from their class to meet with the teams from the other class on several occasions in order to coordinate the project activities. We also arranged multiple video calls with the Marine Biology clients and representatives from all teams. The teams shared a common repository for all of the images and were encouraged to provide access to their codebases through shared GitHub repositories. In the end, from our perspectives as instructors, the collaboration effort was successful in the sense that all teams developed viable proof-of-concept products that can be used by the client. However, there is also room for improvement with respect to the following aspects:

- Division of labor: One of the more tedious aspects of DL with images is the need for labeled sample images. In our case, in addition to a small set of labeled images provided by the clients, all teams added more labeled images. However, they did not communicate well enough to effectively share their labeled sets between the teams. While we as instructors encouraged them to do so, we only realized at the end of the quarter that the teams did not follow up on this.

- Instructor involvement: Although both instructors were very motivated and engaged with the projects, the overall class sizes (35-40 each for all three sections) limited the time they could devote to this. In addition, the Deep Learning class was a new class for that instructor and required considerable time to prepare class material. While graders were used for both classes, they focused on other activities such as lab and homework 
assignments. This allowed the primary instructors to spend more time on the team projects, but one or two extra graders would have been helpful to facilitate better collaboration across teams.

- Logistics: The labs for both classes should meet at same time to allow student communication. The time in which the lab is assigned should be synchronized carefully; in the DL class due to the long introductory labs the collaborative project didn't start until Week 6 while the AI class started much earlier.

\section{Conclusions}

Despite the constraints and difficulties encountered, we considered this experiment a success: all the student teams involved in the collaboration across the two classes learned about different approaches to the same problem, gathered valuable experience about collaboration under serious practical constraints, and became more aware of the need to actively engage with their respective partner teams and with the clients. Although we have no concrete evidence for this, we believe that this led to higher-quality outcomes for those projects, compared with a scenario where they would do the same project without the cross-class collaboration. Especially in the shark teams, there was a spirit of "competitive collaboration" with an informal exchange of current performance values. This was mostly noticeable towards the end of the quarter, where the potential for effective collaboration was limited. We as instructors learned valuable lessons: setting the right conditions for such collaborations, including guidance and clearly defined expectations, is of very high importance.

The particular configuration of the two classes also created an interesting scenario: Can teams with different backgrounds using related methods achieve comparable results? Although the evidence is limited due to the small sample size, the answer seems to be yes: at least one of the two teams in a more general class on Artificial Intelligence using Deep Learning methods and libraries was able to create a proof-of-concept system for shark detection with an overall performance that is within the same range as the team from the class on Deep Learning. Clearly, the team from the Deep Learning class had a better understanding of the underlying methods and more experience in using the respective tools. On the other hand, they spent more time learning about these methods and tools before they started working on the specific shark detection problem.

\section{Future Work}

Due to scheduling constraints, the next iteration of this collaboration will probably take place during the 2020-21 Academic Year. Starting with the logistics, we will try to make arrangements such that there is more overlap between the class times. Most likely there will be projects again involving teams from two AI sections and one DL section, so a perfect overlap will not be possible. Given the room constraints at our institution, it will also be difficult to improve the classroom and lab locations. We will more closely align the schedules for the overall activities in both classes and emphasize preparatory measures like data set provisioning and curation (in particular, labeling images) that can be done early. We are also planning to schedule regular meetings between representatives of the teams involved and the clients. In addition, we are seeking additional funding for student assistants whose responsibility will be to support the collaboration across the teams and with the clients. 


\section{Acknowledgements}

We would like to thank the students involved in this experiment for their openness and positive attitude during their collaboration activities. The data set was provided by the Shark Lab at CSU Long Beach and we gratefully acknowledge the support we received from the shark experts there, in particular the director, Dr. Chris Lowe, and Graduate Student, Patrick Rex.

\section{References}

1. M. LaalSeyed, and M. Ghodsi (2012) "Benefits of collaborative learning" Elsevier Proceedings Social and Behavioral Sciences, Volume 31, Pages 486-490.

2. E.F., Barkley, K.P. Cross, and C.H. Major (2005). Collaborative learning techniques: A handbook for college faculty. San Francisco: Jossey-Bass.

3. D.W Johnson, R. Johnson, and K. Smith (1998). Active learning: Cooperation in the college classroom. Edina, MN: Interaction Book Company.

4. D. Kantor (2010), Four-Player Model of Communication. Kantor's Institute. http://www.yacavone.com/pdf/KantorFourPlayerSummaryV2.pdf. Source: Dialogue and the Art of Thinking Together, by William Isaacs, and The Necessary Revolution, by Peter Senge, et al. Summarized by Michael J. Yacavone 2010.

5. C. Kivunja (2015). Using De Bono's Six Thinking Hats Model to Teach Critical Thinking and Problem- Solving Skills Essential for Success in the 21st Century Economy. Creative Education. 6. 380-391.10.4236/ce.2015. 63037.

6. E. Hammar Chiriac (2014) "Group work as an incentive for learning - students' experiences of groupwork". Frontiers in Psychology v.5 pp.558.

7. M. Pantoja and Z. Wood (2018) "Inter Class Collaboration for Computer Science Classes" ASEEPSW 2018, Boulder, Co.

8. N. Sharma, P. Scully-Power, and M. Blumenstein (2018) Shark Detection from Aerial Imagery Using Region-Based CNN, a Study. In: Mitrovic T., Xue B., Li X. (eds) AI 2018: Advances in Artificial Intelligence. AI 2018. Lecture Notes in Computer Science, vol 11320. Springer, Cham

9. K. Jeremy, M. Johann, G. Kirk, H. Michael R (2016) "Using unmanned aerial vehicles (UAVs) to investigate shark and ray densities in a shallow coral lagoon," in Marine Ecology Progress Series, vol. 560, p. 237-242, November 2016.

10. T. Ahilan, V.Aswin Adityan, S. Kailash (2015) "Efficient Utilization of Unmanned Aerial Vehicles (UAV) for Fishing through Surveillance for Fishermen" in World Academy of Science, Engineering and Technology International Journal of Aerospace and Mechanical Engineering Vol:9, No:8.

11. S. Ren, K. He, R. Girshick and J. Sun (2015) Faster R-CNN: Towards Real-Time Object Detection with Region Proposal Networks. arXiv:1506.01497.

12. R. Girshick (2015) Fast R-CNN. arXiv:1504.08083.

13. K. He, G. Gkioxari, P. Dollr and R. Girshick. Mask R-CNN (2020) Mask R-CNN. IEEE Transactions on Pattern Analysis and Machine Intelligence, 42(2), 386-397. https://doi.org/10.1109/TPAMI.2018.2844175

14. A. Krizhevsky, I. Sutskever and G. E. Hinton (2017) ImageNet Classification with Deep Convolutional Neural Networks. Communications of the ACM, 60(6), 84-90. https://doi.org/10.1145/3065386

15. J. Wang and L. Perez. (2017) The Effectiveness of Data Augmentation in Image Classification using Deep Learning.arXiv:1703.06870, 2017.

16. J. Redmon, S. Divvala, R. Girshick and A. Farhadi (2015) You Only Look Once: Unified, Real-Time Object Detection. arXiv:1506.02640 
17. Abhineet Singh, Marcin Pietrasik, Gabriell Natha, Nehla Ghouaiel, Ken Brizel and Nilanjan Ray (2020) "Animal Detection in Man-made Environments". arXiv:1910.11443; to appear in WACV 2020.

18. S. Rose (2019) Increasing Representation of Underrepresented Groups Among STEM Faculty Members. Higher Education Today from American Council on Education.

19. P. Cantrell , and J. Ewing-Taylor (2013) Exploring STEM Career Options through Collaborative High School Seminars, The Journal of Engineering Education (JEE) https://doi.org/10.1002/j.21689830.2009.tb01026.x

20. C. Alvarado, S. Villazon, and B. Tamer (2019) Evaluating a Scalable Program for Undergraduate CS Research. In Proceedings of the 2019 ACM Conference on International Computing Education Research (ICER '19). Association for Computing Machinery, New York, NY, USA, 269-277. DOI: https://doi.org/10.1145/3291279.3339406

21. L. Barker (2009) Student and Faculty Perceptions of Undergraduate Research Experiences in Computing. ACM Trans. Comput. Educ. 9, 1, Article 5 (March 2009), 28 pages. DOI: https://doi.org/10.1145/1513593.1513598

22. Lopatto D. (2004) Survey of Undergraduate Research Experiences (SURE): first findings. Cell Biol Educ.3(4):270-277. doi:10.1187/cbe.04-07-0045

23. J. Margolis and A. Fisher (2002). Unlocking the clubhouse: Women in computing. MIT press.

24. J. Peckham, P. Stephenson, J.-Y. Hervé, R. Hutt, and M. Encarnação (2007) Increasing student retention in computer science through research programs for undergraduates. In Proceedings of the 38th SIGCSE technical symposium on Computer science education (SIGCSE '07). Association for Computing Machinery, New York, NY, USA, 124-128. DOI:https://doi.org/10.1145/1227310.1227354

25. M. Hewner, and M. Knobelsdorf (2008). Understanding computing stereotypes with selfcategorization theory. In Proceedings of the 8th international Conference on Computing Education Research (pp. 72-75).

26. J. J. Salazar, K. Krein, P. Kramer, G. Chu, A. Rice, M. Pangburn and M. Pantoja (2019). "Shark Detection Using Mask R-CNN," California Polytechnic State University, San Luis Obispo, California, U.S.A., CSC 487 F19, 12/19.

27. R. Kirkpatrick, G. Marquez, C. Virostek, F. Alestrom, and F. J. Kurfess (2019). "Shark/Surfer Spotting," California Polytechnic State University, San Luis Obispo, California, U.S.A., CSC 480 F19 Team 15, 12/19. Accessed: Dec. 11, 2019. [Online]. Available: https://drive.google.com/file/d/1Eknj6sjDr7NmsRBAc2YfQY6ZIC2jpf9R/view?usp=sharing.

28. N. Kyono, S. Rizzo, L. Trujillo, R. Miller, T. Kalvakolanu, A. Erin and F. Kurfess (2019). "Shark Spotting," California Polytechnic State University, San Luis Obispo, California, U.S.A., CSC 480 F19 Team 3, 12/19. Accessed: Dec. 11, 2019. [Online]. Available: https://drive.google.com/file/d/1Eknj6sjDr7NmsRBAc2YfQY6ZIC2jpf9R/view?usp=sharing. 